\title{
Modelagem Magnética em Furos para Avaliação do Comprimento de Estacas
}

\author{
Vicente Luiz Galli (IPT) \\ Copyright 2021, SBGf - Sociedade Brasileira de Geofísica \\ This paper was prepared for presentation during the $17^{\text {th }}$ International Congress of the \\ Brazilian Geophysical Society, online event, Brazil, 8-11 November 2021. \\ Contents of this paper were reviewed by the Technical Committee of the $17^{\text {th }}$ \\ International Congress of the Brazilian Geophysical Society and do not necessarily \\ represent any position of the SBGf, its officers or members. Electronic reproduction or \\ storage of any part of this paper for commercial purposes without the written consen \\ of the Brazilian Geophysical Society is prohibited.
}

\section{Abstract}

Forward modeling and processing for borehole magnetic data were developed to estimate de depth of a pile. By applying the pseudo-gravity transformation to synthetic data calculated for magnetic induction in a pile in borehole geometry, it is verified that magnetic anomalies become easy to interpret and the depth of a pile is effectively resolved. The applicability for field data was performed successfully.

\section{Introdução}

A avaliação do comprimento de estacas em estruturas em subsuperfície é de fundamental importância no campo da engenharia civil. Existem alguns métodos para esse propósito, como o método sísmico paralelo, a perfilagem eletromagnética, a resistividade elétrica entre outros. Uma fundação é constituída de concreto e aço e este por sua vez gera campos magnéticos induzidos em resposta ao campo geomagnético da Terra. Neste contexto, com intuito de aprimorar o uso de ensaios magnéticos em furos de sondagem para estimar a profundidade de estacas em estruturas em subsuperfície, realizou-se modelamento magnético utilizando modelos sintéticos de estacas em diferentes cenários. Por meio da transformação da anomalia magnética modelada em anomalia pseudo-gravimétrica seguido do gradiente horizontal pôde-se identificar os limites da estaca e avaliar o seu comprimento com precisão.

\section{Modelamento}

Foram feitas simulações de levantamentos magnéticos, utilizando-se como fontes causadoras das anomalias modelos digitais idealizados com fundações constituídas de materiais com susceptibilidades magnéticas distintas em relação ao solo. O modelo digital foi composto por um elemento de fundação (estaca) com aço em sua estrutura, cravada no solo. Esse modelo digital foi montado com a finalidade de obter as respostas típicas a serem obtidas nos ensaios geofísicos de magnetometria associadas aos elementos de uma fundação hipotética 3D para diferentes cenários. A partir do modelo digital foram gerados mapas e perfis magnéticos com a finalidade de estabelecer os aspectos que devem ser observados, relacionados às respostas obtidas pelo método magnético, quando o alvo investigado for um elemento de fundação. Para essas simulações utilizou-se o software MAG3D. Os dados magnéticos gerados a partir do modelo digital teórico foram visualizados por meio do software Oasis.

A Figura 1 mostra o modelo sintético de fundação construído com uma estaca; posicionada entre $9 \mathrm{~m}$ e 10 $\mathrm{m}$ no eixo-x, com $1 \mathrm{~m}$ de largura; entre $9 \mathrm{~m}$ e $10 \mathrm{~m}$ no eixo-y, com $1 \mathrm{~m}$ de espessura; e entre $0 \mathrm{~m}$ e $-5 \mathrm{~m}$ no eixo-z, com comprimento de $5 \mathrm{~m}$. O valor de susceptibilidade magnética considerado para o material usado na simulação foi ferro $(k=300000)$ e solo $(k=$ $\left.1 \times 10^{-5}\right)$.

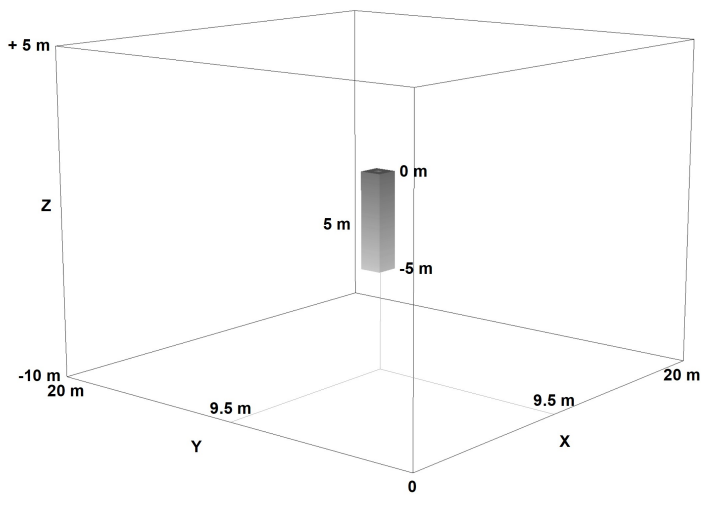

Figura 1 - Modelo de fundação constituída por uma estaca com aço representada por bloco retangular na cor cinza, com 5 metros de comprimento, $1 \mathrm{~m}$ de largura e 1 $m$ de espessura.

A Figura 2 apresenta dois mapas magnéticos gerados a partir do modelo ilustrado na Figura 1, um a 3 metros acima da superfície, ou seja, a 3 metros acima do topo da estaca do modelo; e outro a 8 metros abaixo da superfície, isto é, a 3 metros abaixo da base da estaca do modelo. 


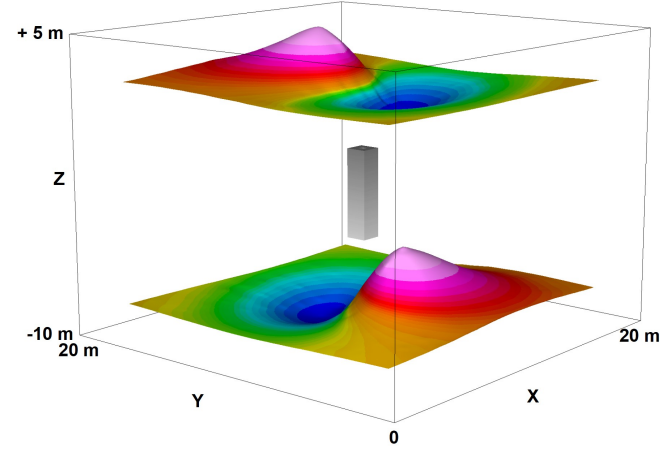

Figura 2 - Visualização $3 D$ de dois mapas magnéticos gerados a partir do modelo digital, um a 3 metros acima do topo da estaca do modelo; e outro a 3 metros abaixo da base da estaca do modelo. A cor lilás representa valores altos do campo magnético total e a cor azul corresponde a valores baixos do campo magnético total. O bloco retangular na cor cinza representa o modelo de estaca. Inclinação magnética $i=-37$ graus e declinação magnética $D=-21$ graus.

A segunda simulação foi idealizada para o mesmo modelo de fundação. O campo magnético anômalo foi gerado ao longo de 12 furos de sondagens posicionados ao redor da estaca. A Figura 3 mostra, em planta, a posição dos 12 furos de sondagens em relação à estaca e as Figuras 4 e 5 apresentam em 3D o campo magnético anômalo gerado com cores variando de azul a lilás que representam as variações da intensidade do campo magnético total que ocorrem devido ao aço presente na estaca de fundação do modelo hipotético.

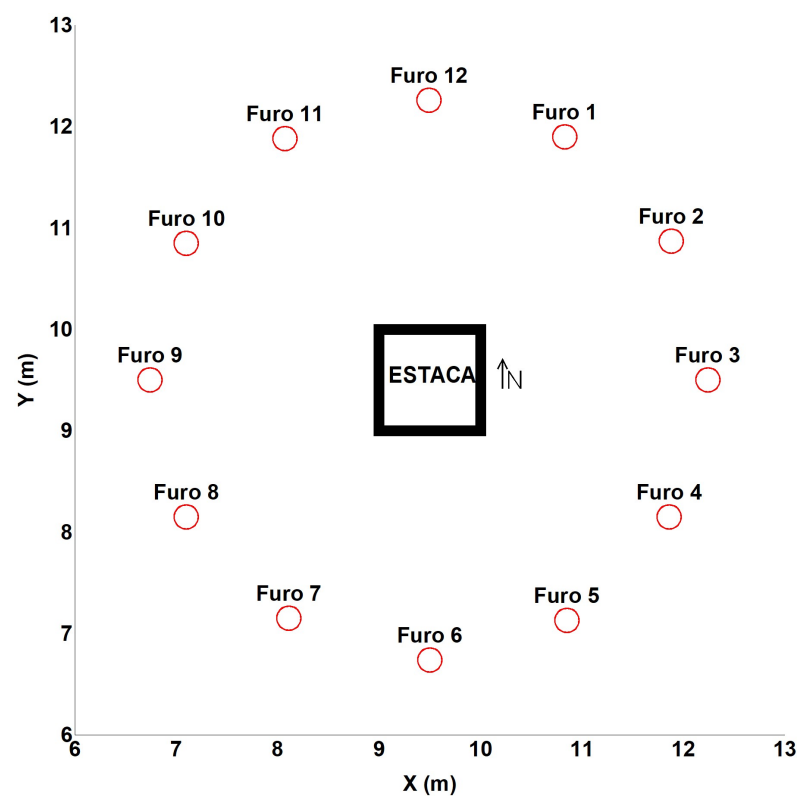

Figura 3 - Posição, em planta, dos 12 furos ao redor da estaca hipotética do modelo em que o campo magnético anômalo foi gerado.

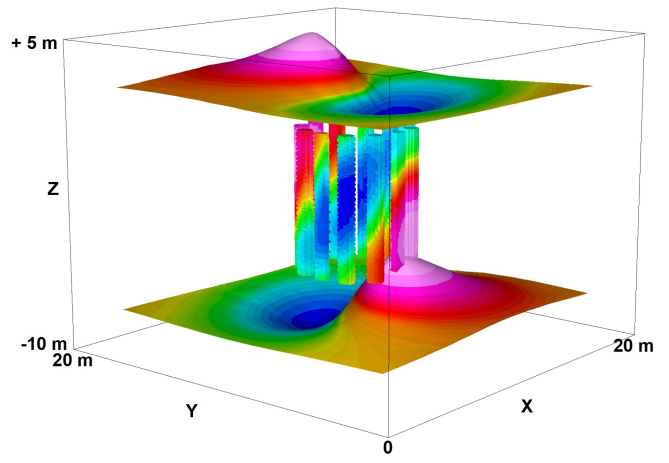

Figura 4 - Visualização $3 D$ do campo magnético anômalo calculado ao longo de 12 furos de sondagens posicionados ao redor da estaca hipotética e dois mapas magnéticos, um a 3 metros acima do topo da estaca do modelo; outro a 3 metros abaixo da base da estaca do modelo. A cor lilás representa valores altos do campo magnético total e a cor azul corresponde a valores baixos do campo magnético total. Inclinação magnética i=-37 graus e declinação magnética $D=-21$ graus.

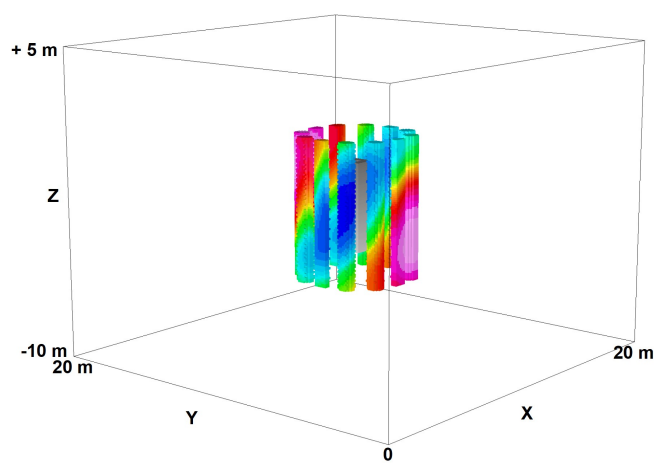

Figura 5 - Visualização $3 D$ do campo magnético anômalo gerado ao longo de 12 furos de sondagens posicionados ao redor da estaca hipotética. A cor lilás representa valores altos do campo magnético total e a cor azul corresponde a valores baixos do campo magnético total. Inclinação magnética $i=-37$ graus $e$ declinação magnética $D=-21$ graus.

Nota-se nessa figura 5 que os dados obtidos na simulação para esses furos não apresentam qualquer evidência sobre os limites da estaca. Assim, adentrou-se no domínio da matemática na busca de um processamento que possa ser aplicado ao dado magnético com intuito de desvendar os limites da estaca e avaliar o seu comprimento.

\section{Processamento}

O processamento dos dados magnéticos focou delimitar os limites do elemento de fundação, isto é, o início e o fim da estaca e assim avaliar o seu comprimento. 
Partiu-se do princípio de que:

a) uma anomalia gravimétrica hipotética sobre uma fonte pode ser calculada a partir de uma anomalia magnética, por meio da transformação pseudo-gravimétrica (Baranov, 1957, Geophysics v.22, p.359-383); e

b) o gradiente horizontal máximo de uma anomalia gravitacional está localizado quase sobre as bordas (limite) das fontes tabulares (Cordell, 1979 and Cordell and Grauch, 1985, Soc. Explor. Geophys. Spec. Vol., p.181-197, and Blakely and Simpson, 1986, Geophysics, v. 51, p. 1494-1498)

\section{TRANSFORMAÇÃO PSEUDO-GRAVIMÉTRICA}

A relação de Poisson afirma que a anomalia magnética sobre fontes magnéticas pode ser transformada na anomalia gravitacional que seria esperada se a distribuição da magnetização fosse igual à distribuição da densidade. Esta transformação pseudo-gravimétrica é realizada como uma operação de filtragem no domínio da frequência (transformadas de Fourier), um filtro que depende da direção da magnetização e da direção do campo terrestre.

Inicialmente, o perfil magnético gerado para cada um dos furos foi transformado no perfil pseudo-gravimétrico; em seguida, ao perfil pseudo-gravimétrico resultante foi aplicado o gradiente horizontal. Nesta etapa, usou-se o software MagPick.

A Figura 6 apresenta em 3D o resultado do processamento aplicado aos perfis magnéticos gerados para os 12 furos ao redor da estaca do modelo. Nota-se nesta figura que, independente do lugar em que o furo tenha sido feito em relação à estaca, o gradiente máximo pseudo-gravimétrico coincide com os limites da estaca, isto é, o início e o fim da estaca, podendo-se avaliar o seu comprimento.

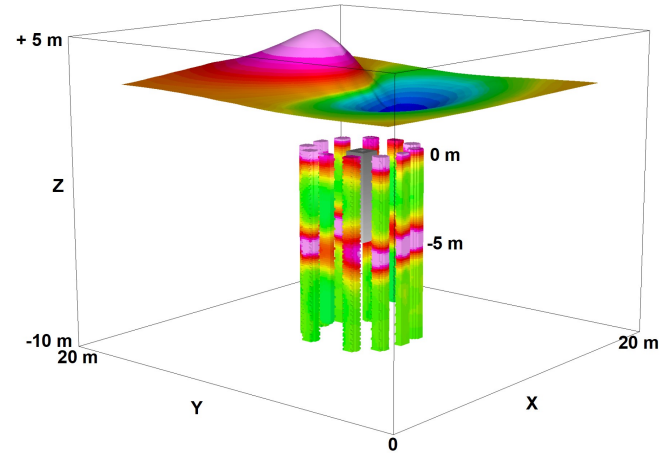

Figura 6 - Visualização $3 D$ do gradiente pseudogravimétrico dos 12 furos posicionados ao redor da estaca hipotética e do mapa magnético do campo total gerado a 3 metros acima da superfície. A cor lilás representa valores máximos do gradiente e coincidem com os limites da estaca do modelo representado na figura pelo bloco retangular na cor cinza. Inclinação magnética $i=-37$ graus e declinação magnética $D=-21$ graus.

\section{Conclusões}

Das simulações realizadas utilizando-se um modelo digital composto por um elemento de fundação (estaca) cravada no solo com aço em sua estrutura, conclui-se que a transformação pseudo-gravimétrica, seguida do gradiente horizontal é um processamento factível de se aplicar a dados magnéticos realizados em furos para avaliar o comprimento de estacas de fundação com armadura de aço em sua estrutura.

\section{Referências Bibliográficas}

Baranov, V., 1957. A new method for interpretation of aeromagnetic maps: pseudo-gravimetric anomalies. Geophysics v.22, p.359-383.

Beattie, G. J. (1982). "Pile Length Determination Induction Field Method." Technical Note 5-3, Works Consultancy Services, Central Laboratories, Lower Hutt, New Zealand.

Blakely, R. J., and R. W. Simpson, 1986, Approximating edges of source bodies from magnetic or gravity anomalies: Geophysics, 51, 1494-1498.

Cordell, L., 1979, Gravimetric expression of graben faulting in Santa Fe County and the Espanola Basin, New Mexico, in R. V. Ingersoll, ed., Guidebook to Santa Fe County: 30th Field Conference, New Mexico Geological Society, 59-64.

Cordell, L., and V. J. S. Grauch, 1985, Mapping basement magnetization zones from aeromagnetic data in the San Juan Basin New Mexico, in W. J. Hinze, ed., Utility of regional gravity and magnetic maps: SEG, 181-197.

Hiltunen, D.R. and Roth, M.J.S. (2003). "Investigation of Bridge Foundation Sites in Karst Terrane via MultriElectrode Electrical Resistivity." 2003 Highway Geophysics NDE Conference. Orlando, FL.

Hossain, M. S., Khan, M. S., Hossain, J., Kibria, G., and Taufiq, T. (2011). "Evaluation of Unknown Foundation Depth using Different NDT Method." Journal of Performance of Constructed Facilities, Vol. 27, No. 2, 209-214.

Jalinoos, F., Gibson, A., Diehl, J., Hadfield, P. and Gordon, G. (2006). "Determination of Unknown Length of Sheet Piles Using Three Geophysical Logging Methods" 2006 Highway Geophysics NDE Conference, St. Louis, MO.

Jo, C.H., Cha, Y.H., and Choi, J.H. (2003). "A Borehole Magnetic Logging Tool for Estimating Unknown Foundation Depths." 2003 Highway Geophysics NDE Conference, Orlando, FL.

Khan, M.S. Hossain, M.S., Hossain, J., and Kibria, G. (2012). "Determining Unknown Bridge Foundation Depth by Resistivity Imaging Method." Proceedings Geo-Congress 2012, Oakland, CA.

Sack, D.A., and Olson, L.D. (2009). "Combined Parallel Seismic and Cone Penetrometer Testing of Existing Foundations for Foundation Length and Evaluation" Proceedings 2009 International Foundation Congress and Equipment Expo, Orlando, FL. 